\title{
Abstracts der Posterausstellung
}

\section{ROSE-Studie \\ Lachmann A \\ Hamburg}

Ein bedeutender Anteil von Opiatabhängigen hat keinen Kontakt zum Suchthilfesystem.

Der Hintergrund dieser Studie ist, dass Opiatabhängige, die sich nich in Behandlung befinden oder nicht ausreichend vom bestehenden Hilfesystem profitieren, ein weitaus höheres Risiko für somatische und psychiatrische Komorbidität, für Drogennot- oder -todesfälle und riskante Konsummuster und delinquentes Verhalten aufweisen als integrierte Konsumenten.

Ziel der Studie ist es, epidemiologische Daten der o. g. Zielgruppe auf europäischer Ebene zu erheben und daraus Handlungsleitlinien und Empfehlungen für eine verbesserte Versorgung abzuleiten.

Das Projekt wird in Zusammenarbeit mit weiteren neun europäischen Zentren durchgeführt. Weitere Ziele:

- Erhebung der Prävalenzraten von Opiatabhängigen, die sich nicht in Behandlung befinden und nicht erfolgreich im bestehenden Suchthilfesystem integriert sind

- Beschreibung der beiden Zielgruppen im Hinblick auf somatische und psychiatrische Morbidität

- Beschreibung und Evaluation vorhandener Interventionsstrategien für die beiden Zielgruppen vor dem Hintergrund der unterschiedlichen nationalen Gegebenheiten und Settings

- Erhebung der Behandlungsbedarfe und -bedürfnisse der Opiatabhängigen, die sich in keiner suchttherapeutischen Behandlung befinden

- $\quad$ Entwicklung von Standards, Guidelines und zukünftigen Anforderungen für die beiden Zielgruppen im Hinblick auf Europa.

Die Methodik der Studie umfasst sowohl quantitative als auch qualitative Erhebungsverfahren. Aus der Zielgruppe der nicht erreichten Opiatabhängigen werden 50 Personen und aus der Gruppe der nicht adäquat Behandelten 100 Personen befragt. Weiterhin werden Experten aus dem Hilfesystem, Drogenkoordinatoren, Mitarbeiter der Polizei, substituierende Ärzte und Mitarbeiter aus dem Strafvollzugssystem an der Befragung teilnehmen. Eine Re-Analyse vorhandener Forschungsberichte zu epidemiologischen Daten ist ebenfalls Bestandteil der Untersuchung. re:job - medizinische und berufliche Rehabilitation

für Opioidabhängige

Lojewski I, Vitt J, Karg K, Gier C, Kazich-Lösky U, Kumbier-Jordan B Göttingen

Nach einer Modellphase ist re:job seit dem Jahr 2002 Regelangebot der LVA Hannover zur medizinischen und beruflichen Rehabilitation opioidabhängiger Menschen, die sich in einer Substiutionsbehandlung befinden oder an einer medikamentös gestützten Abstinenztherapie teilnehmen.

In der systematischen Eingangs- und Verlaufsdiagnostik erfolgen medizinische, sozialtherapeutische und arbeitstherapeutische Beurteilungen, einschließlich standardisierter Verfahren, sowie die Selbsteinschätzung des Patienten, die in einem individuellen Rehabilitationsplan dokumentiert werden.

Unter der Fragestellung, inwieweit es gelingt, die Rehabilitanden auf den ersten Arbeitsmarkt zu reintegrieren und welche Faktoren dies begünstigen, wird re:job wissenschaftlich evaluiert.

Bei den Rehabilitanden zeigt sich ein hohes Maß an psychiatrischer und körperlicher Komorbidität mit verminderter Belastungsfähigkeit, sozialen Problemen und defizitären Grundarbeitsfähigkeiten. Im Gegensatz dazu stehen gute fachpraktische Fähigkeiten, arbeitsbezogene Sozialkompetenz sowie Verantwortungsbereitschaft. Besonders Patienten mit der Doppeldiagnose „Psychose und Sucht“ profitieren von der interdisziplinären Behandlung im teilstationären Rahmen.

Erwerbstätigkeit stellt einen wesentlichen Faktor für die physische, psychische und soziale Gesundheit dar, d.h., eine ganzheitliche Rehabilitation muss auch die Integration in gesellschaftliche und berufliche Zusammenhänge und damit den Zugang zur Erwerbstätigkeit beinhalten. Das vorgestellte Rehabilitationsangebot findet eine breite Akzeptanz und hat bei freiwilliger Teilnahme eine hohe Haltequote. Bisherige Erfahrungen mit dem hier vorgestellten Rehabilitationskonzept zeigen die Verbesserung der psychischen und körperlichen Gesundheit und der sozialen Integration. Durch den Aufbau realistischer Zukunftsperspektiven und die Förderung der Selbstwirksamkeitserwartungen wird die Motivation zu Eigeninitiative und abstinentem Selbstkonzept unterstützt. Es bestätigt sich die (Re-)Integrierbarkeit substituierter Drogenabhängiger auf den ersten Arbeitsmarkt. 
Gruppenmusiktherapie bei drogenabhängigen Jugendlichen mit psychotischem Erleben

Becker T, Thoms E

Leipzig

Im Rahmen der multiprofessionellen Behandlung von drogenabhängigen Kindern und Jugendlichen auf der Therapiestation Teen Spirit Island Leipzig wird das Modul Musiktherapie in Bezug auf Veränderungen des Sozialverhaltens bei Psychoseerkrankten untersucht Durch Einsatz des Mediums Musik ist es möglich, Veränderungen im Beziehungsaufbau und in Beziehungsgestaltung, Kontaktverhalten, in Wahrnehmung und Grundstimmung zu erzielen. Durch musiktherapeutische Interventionen können Therapieinhalte und -ziele beschrieben und erreicht werden. Musik spielt als Transporteur der Auseinandersetzung mit sich selbst und dem sozialen Gegenüber eine entscheidende Rolle und kann als gruppentherapeutisches Element bei psychotisch erkrankten drogenabhängigen Jugendlichen genutzt werden.

Praxis- und forschungsrelevante Ergebnisse einer

Basisdatenerhebung an stationär behandelten

drogenabhängigen Kindern und Jugendlichen eines

Behandlungsjahrgangs

Thoms E, Petros S

Leipzig

Das Poster stellt eine Retrospektivstudie über einen Behandlungsjahrgang der ersten Station für abhängigkeitskranke Kinder und Jugendliche in den neuen Bundesländern vor. Aus der Analyse aller Einzelfaktoren werden interessante Aspekte dargestellt, die möglicherweise zum Konsum psychotroper Substanzen beitrugen und Ursachen darstellen, die zum vorzeitigen Abbruch der Therapie führten. Auffällig ist die hohe Zahl an Patienten mit Leistungsstörungen. Auch aus dieser Studie wird deutlich, dass möglicherweise unbehandelte ADHS-Patienten zu Suchtpatienten werden. Familiäre Verhältnisse und psychische Erkrankungen innerhalb der Familien tragen zu einer Suchtentwicklung entscheidend bei. Das Rauchen stellt sich als suchtbahnender und suchtaufrechterhaltender Faktor dar. In der Behandlung von drogenabhängigen Kindern und Jugendlichen ist ein besonderer therapeutischer Rahmen notwendig und auch der Einsatz von effektiven Psychopharmaka ist im Entzugsmanagement erforderlich.

Komorbide Störungen bei suchtmittelabhängigen Kindern und Jugendlichen Retrospektivanalyse von 745 in der Klinik für Kinder- und Jugendpsychiatrie, Park-Krankenhaus, Leipzig, in den Jahren 1998 bis 2001 stationär behandelten $\mathrm{Pa}$ tienten im Vergleich zu 159 im Jahr 2002 behandelten Patienten.

Thoms $E$

Leipzig

In Leipzig wird seit 1998 die stationäre Behandlung suchtmittelabhängiger Kinder und Jugendlicher in der Klinik für Kinderund Jugendpsychiatrie in einem zweiphasigen Therapiesetting durchgeführt.

Dargestellt werden die Ergebnisse der umfangreichen retrospektiven Patientenanalyse der behandelten minderjährigen Suchtmittelabhängigen auf der Therapiestation für drogenabhängige Kinder und Jugendliche, TEEN SPIRIT ISLAND - LEIPZIG, aus den Jahren 1998 bis 2001 und dem Jahr 2002.

Eine komorbide psychiatrische Zusatzdiagnose hatten in der ersten Analyse insgesamt 68\% der behandelten Patienten. Im Jahr 2002 ist durch eine Verlängerung der Verweildauer und eine dadurch bedingte intensivere Psychodiagnostik die psychiatrische Zusatzdiagnose bei $86 \%$ der Patienten festgestellt worden. Die Zweitdiagnose Psychose trat von 1998-2001 in 39 Fällen auf, im Jahre 2002 in 6 Fällen.
Insgesamt ist die Behandlungsklientel im Altersdurchschnitt jünger geworden, die Anzahl der weiblichen Patienten hat zugenommen, die Patienten werden früher vom Hilfesystem erreicht und das Konsummuster hat sich in Richtung Cannabis-, Ecstasy- und Crystalabhängigkeit verschoben

Drogenabhängige minderjährige Schwangere - der innere Konflikt zwischen Abbruch und Kinderwunsch

Thoms $E$

Leipzig

Drogensüchtige minderjährige Schwangere stellen eine äußerst problematische Gruppe der schwangeren Minderjährigen dar. Das Rauscherleben erzeugt eine intrapsychische Regression und führt letztendlich auch durch die Aufrechterhaltung der Sucht zu einer strukturellen Störung. Auf dem Boden dieser psychischen Empfindlichkeit wird eine Schwangerschaft von dem Mädchen oder der Jugendlichen oft als Chance gesehen, dass die Sehnsucht, das eigene Leben noch mal leben zu können, erfüllt wird. Von außen betrachtet wird die Situation eher mit dem Ausdruck ,sie ist noch selber ein halbes Kind“ beschrieben. Gründe der frühen Schwangerschaft sind oft negative sexuelle Erfahrungen, Gewalt, Missbrauch und auch der Verlust altersgemäßer sexueller Bedürfnisse. Der Umgang mit Verhütung ist oft problematisch, besonders wenn Erfahrungen mit Prostitution bestehen. Die Schwangerschaft und die erhoffte Geburt des Kindes werden verbunden mit einem Neubeginn. Die Väter sind oft instabile Persönlichkeiten, reagieren häufig mit Ablehnung (70\%) oder Idealisierung. Vor diesem Hintergrund entsteht sowohl für die Jugendlichen, für die Eltern als auch für die Hilfesysteme ein erheblicher Konflikt in der Beratung bezüglich Geburt oder Abbruch. Die Bearbeitung dieses Konfliktes führt regelmäßig zur Verwicklung zwischen den einzelnen Beteiligten, und nur die Bearbeitung dieses Konfliktes mit den Jugendlichen, den Eltern und den Hilfesystemen ermöglicht die Planung adäquater Hilfen.

Teen Spirit Island - Leipzig Therapiestation für drogenabhängige Kinder und Jugendliche

Fromme E, Thoms

Leipzig

Die Reduktion von Schnittstellen ist für die beziehungsgestörten drogenabhängigen Kinder und Jugendlichen eine primäre konzeptionelle Aufgabe. Durch die Vernetzung der Therapiestation mit den komplementären Einrichtungen, der vom Therapeutenteam durchgeführten Drogensprechstunde im Jugendhaus, der tagesklinischen Behandlung, der integrierten Nachsorge und der Zusammenarbeit mit komplementären Jugendhilfeeinrichtungen wird ein jugendgerechtes Setting geschaffen.

Früh traumatisierte Kinder und Jugendliche können durch maximale Beziehungskonstanz besser ihre intrapsychische Problematik bearbeiten. Das multiprofessionelle Team benutzt die verschiedenen kinder- und jugendpsychiatrischen Standardmodule besonders mit dem Schwerpunkt der Eigenbeteiligung von Patienten am Stationsalltag und der besonderen freizeittherapeutischen Aktivität von Climbing, Beachvolleyball und Schwimmen, um die Patienten qualitativ hochwertig behandeln zu können.

Versorgungsstrukturen für drogenabhängige Kinder und Jugendliche in Kliniken für Kinder- und Jugendpsychiatrie und Psychotherapie in Deutschland

Thoms $E$

Leipzig

Seit 1990 wird das Thema Drogenabhängigkeit zunehmend auch von der Kinder- und Jugendpsychiatrie als originäres Arbeitsfeld wahrgenommen. Eine besondere Herausforderung stellt die Entwicklung zielgerichteter Konzepte und vernetzter Versorgungsstrukturen von 
Jugendhilfe, Drogenhilfe, Schule, Rehabilitation und Kinder- und Jugendpsychiatrie dar. In den letzten Jahren sind einige spezialisierte Behandlungsstationen für diese Patientengruppen entstanden. Das Behandlungskonzept unterscheidet sich von den Versorgungsstrukturen für Erwachsene. In Deutschland bestehen allerdings immer noch erhebliche Versorgungsdefizite.

Epidemiologische Analyse der Alkoholentzugsfälle in Berliner Krankenhäusern

Sieber E, Binting S, Willich SN

Berlin

Hintergrund: Seit 1993 werden alle Behandlungsfälle in den Krankenhäusern Berlins diagnosespezifisch erfasst. Mit dem Klassifikationswechsel (2000) bei der Verschlüsselung der Hauptdiagnose von der ICD 9 auf die ICD10 tritt ein Bruch in der Datenkontinuität auf, weswegen erst die Daten des Jahres 2001 in die Zeitreihe einbezogen werden.

Zielstellung: Die Dimension des Problems der Alkoholabhängigkeit in der Diagnosestruktur aller 74 (1993) bzw. 70 (2001) Berliner Krankenhäuser und die Entwicklung der zentral erfassten Parameter werden dargestellt.

Material und Methoden: Die Analyse umfasst alle Fälle mit den Hauptdiagnosen Alkoholismus (303 ICD9) und Alkoholpsychosen (291 ICD9) von 1993-1999 und für das Jahr 2001 alle Fälle mit Störungen durch Alkohol (F10 ICD10). Diese Datensätze wurden vom Statistischen Landesamt Berlin zur Verfügung gestellt. Unsere Berechnung relativer Häufigkeiten erfolgt durch Bezug auf die mittlere Bevölkerung der jeweiligen Berliner Region.

Ergebnisse: In den Berliner Krankenhäusern gehört die Alkoholabhängigkeit zu den fünf häufigsten Behandlungsursachen insgesamt. Die Fallhäufigkeit startet 1993 mit 289, hat 1997 das Maximum mit 331, liegt 1999 bei 328 und erreicht 2001 nur 197 Fälle je 100000 der Bevölkerung. In den 12 Berliner Stadtbezirken zeigen sich große Unterschiede in den Fallhäufigkeiten (2001: Friedrichshain/Kreuzberg 297 vs. Steglitz/Zehlendorf 109). Das Verhältnis von männlichen zu weiblichen Patienten beträgt 4:1. Die altersspezifischen Fällhäufigkeiten sind am höchsten in der Altersgruppe von 40 bis unter 50 Jahren: bei Männern 744, bei Frauen 187 Fälle je 100000 dieser Altersgruppe (2001). Die durchschnittliche Verweildauer im Krankenhaus sank von 22 Tagen auf 13 Tage. Die meisten Behandlungsfälle (ca. 88\%) liegen in den Fachabteilungen Psychiatrie, Suchtkrankheiten und Innere Medizin.

Schlussfolgerungen: Diese Routinedaten des Krankenhausbereichs können genutzt werden, um Behandlungsstrategien bei Alkoholabhängigkeit bzw. Störungen durch Alkohol zu hinterfragen.
Buprenorphineinstellung/-umstellung und anschließende Dosisreduktion bei schwangeren Opiatabhängigen Siedentopf J-P, Nagel M, Casteleyn S, Dudenhausen J W Berlin

Einleitung: In der Infektionsambulanz der Klinik für Geburtsmedizin der Charité werden jährlich über 40 drogenabhängige Schwangere betreut. Seit Juni 2000 nutzen wir Buprenorphin als Substitutionsmedikament zu einer Erweiterung unseres therapeutischen Spektrums.

Therapiekonzept: Wir bieten perspektivisch jeder Patientin bereits beim Erstgespräch die Umstellung auf BUP im Schwangerschaftsverlauf an. Inwieweit diese Umstellung dann tatsächlich zu realisieren ist, entscheidet der weitere Verlauf von Schwangerschaft und Therapie. Schwangeren Patientinnen, die nicht substituiert werden und im Wesentlichen Heroin konsumieren, bieten wir eine baldige Einstellung auf BUP an.

Zur Durchführung der BUP-Einstellung in der Schwangerschaft halten wir einen stationären Aufenthalt von 5 bis 7 Tagen für angeraten, um eine stabile Substitutionsdosis zu erreichen und mögliche Komplikationen rechtzeitig erkennen und behandeln zu können. Der Umoder Einstellung der Patientin auf BUP kann sich eine ambulante oder stationäre Reduktion anschließen.

Ergebnisse: Von den 114 seit Beginn der Buprenorphinanwendung im Juni 2000 an der Klinik für Geburtsmedizin der Charité betreuten opiatabhängigen Schwangeren wurden 27 Patientinnen im Schwangerschaftsverlauf von uns auf Buprenorphin eingestellt. 6 weitere schwangere Patientinnen waren bei der Erstvorstellung bereits mit BUP substituiert. Insgesamt 8 Patientinnen derjenigen Patientinnen, die bei uns auf Buprenorphin umgestellt wurden, brachen die Buprenorphineinnahme bis zur Entbindung wieder ab.

Schlussfolgerungen: Eine Buprenorphinumstellung in der Schwangerschaft gelingt unter Beachtung einiger Vorsichtsmaßnahmen problemlos und kann neben der stabilen Substitution - die entsprechende Motivation und ein engmaschiges psychosoziales Helfernetz vorausgesetzt - zum erfolgreichen BUP-gestützten Opiatentzug in der Schwangerschaft führen.

Auf dem Poster werden Dosisempfehlungen, Ergebnisse und geburtshilfliche, suchtmedizinische und psychosoziale Kontraindikationen dargestellt.

\section{7}


Gellert, R., Schneider, G.: Substitution und Heroin - Ein Ratgeber für Betroffene, Angehörige und professionelle Helfer. 2002, 144 S. Lambertus, Freiburg im Breisgau. EUR 8,-; ISBN 3-7841-1430-X

Mit diesem Buch legen die Autoren eine handliche Einführung in die Substitutionsbehandlung vor. Die Autoren verzichten auf die medizinische Fachterminologie. Das führt aber in keiner Weise zu geringerer Klarheit und Genauigkeit der Aussagen - im Gegenteil.

Dass 30 von 144 Seiten den Formalien gewidmet werden (müssen), ist nicht Schuld der Autoren, sondern zeigt, wie kompliziert diese Behandlungsform geregelt ist. Wegen des raschen Wechsels der Verordnungen und Richtlinien ist es kaum möglich, die aktuellsten Vorschriften zu erwähnen: Bereits wenige Monate nach Erscheinen war der Abschnitt zur Finanzierung der Behandlung durch die Krankenkassen veraltet. Der mehrseitige Behandlungsvertrag zeigt, wie weit die Substitutionsbehandlung noch immer von einer normalen ärztlichen Behandlung einer chronischen Krankheit entfernt ist: Nicht alle Punkte dieses Vertrages sind durch das spezielle Verhalten einiger Heroinabhängiger zu begründen. Die Forderung, die Übernahme der Kosten anderer Substitutionsmedikamente als Methadon bei der Krankenkasse zu beantragen, ist sicher falsch und nur als Folge der Regressdrohungen einiger Kassenärztlicher Vereinigungen zu verstehen.
Man erkennt in jedem Satz die große Erfahrung der Autoren mit der Substitutionsbehandlung: bei der Bewertung der Substitutions- und der Entwöhnungsbehandlung nicht als konkurrierende, sondern als sich ergänzende Therapieformen, der Bewertung der Urinkontrollen, dem Nutzen der psychosozialen Begleitung, der Warnung vor übereiltem Behandlungsabbruch, der zusätzlichen Verordnung von Psychopharmaka, der kritischen Bewertung von Benzodiazepinverordnungen. Knapp und klar sind Beschreibung der wichtigsten körperlichen und seelischen Begleiterkrankungen inklusive der häufigen Schlafstörungen und der Bemerkungen zur Behandlung schwerer Schmerzen. Vermisst habe ich nur einen kurzen Hinweis auf die wichtigsten Wechselwirkungen von Methadon mit anderen häufig eingesetzten Medikamenten.

Genauso gut sind auch die Hinweise aus dem psychosozialen Bereich: auf die stabilisierende Funktion der Arbeit, die Bedingungen für einen Führerschein, über Schuldenregulierung und Urlaubsplanung.

Dieses Buch ist wegen seiner klaren Sprache für medizinisch nicht vorgebildete Menschen gut verständlich. Es ist auch für Ärzte zur Einführung in die Substitutionsbehandlung geeignet. Wichtig ist der Hinweis der Autoren, dass der Austausch mit anderen substituierenden Kolleginnen und Kollegen hilfreich (ich meine: notwendig) ist.

\section{Tagung}

"Die subjektive Seite der Schizophrenie“

vom 25.-27. Februar 2004

Tagungsort: Universität Hamburg, Hauptgebäude, Edmund-Siemers-Allee 1, 20146 Hamburg

Veranstalter: Prof. Dr. Dieter Naber, Prof. Dr. Michael Krausz

\section{Psychosen emotional betrachtet - Der affektive Anteil an Prozess und Behandlung}

Schwerpunktthemen:

* Subjektive Seite affektiver Psychosen

* Therapie mit viel Emotion

Schizophrenie als affektive Störung? Theoretische und klinische Konzepte

zum Zusammenhang von Denken und Fühlen

* Symptom, Syndrom, Emotion

Anmeldung und Information:

Universitätsklinikum Hamburg-Eppendorf, Klinik für Psychiatrie und Psychotherapie

Kongressbüro Christa Parchmann, Martinistr. 52, 20246 Hamburg

Tel./Fax: 0 40/4 $2803-4804$

E-mail:parchman@uke.uni-hamburg.de

Internet: www.zis-hamburg.de 